\title{
Sífilis: Prevalência num Hospital de Lisboa
}

\section{Syphilis: Prevalence in a Hospital in Lisbon}

\author{
Leonor LOPES $\rrbracket^{1}$, Rita FERRO-RODRIGUES ${ }^{2}$, Samuel LLOBET ${ }^{3}$, Luís LITO ${ }^{3}$, João BORGES-COSTA ${ }^{1,4,5}$ \\ Acta Med Port 2016 Jan;29(1):52-55
}

RESUMO

Introdução: A sífilis é uma doença de transmissão sexual e vertical. A sua incidência está a aumentar na Europa, particularmente em Portugal.

Material e Métodos: Estudo retrospetivo baseado na análise laboratorial de testes treponémicos positivos, entre janeiro e dezembro de 2013, no Hospital de Santa Maria. Foram incluídos doentes internados, da consulta externa, do hospital dia e da urgência. Procedeu-se a caraterização epidemiológica, classificação da doença e de fatores de risco associados.

Resultados: Obteve-se uma amostra de 484 doentes, após exclusão de 51 por ausência de dados clínicos nos processos e de 45 por valores falsos positivos. Verificou-se predomínio do sexo masculino (75\%) e idade média de 47 anos. A maioria (59\%) tinha testes serológicos compatíveis com sífilis no passado e 3,7\% encontrava-se em vigilância clínica. Diagnosticou-se sífilis primária em 13 doentes, secundária em 71, latente precoce em 40, latente indeterminada em 49 e latente tardia em cinco. No grupo sífilis recente, $42 \%(n=124)$ eram seropositivos para o VIH e $8 \%$ tiveram, em simultâneo, este diagnóstico.

Discussão: Salienta-se a elevada prevalência da coinfeção pelo VIH nos doentes com sífilis recente, reforçando a importância de promover a utilização de medidas preventivas. Registaram-se $11 \%$ de formas clínicas tardias, que são de notificação obrigatória desde junho de 2014. Todos os testes serológicos para o diagnóstico de sífilis apresentam limitações, o que enfatiza a importância da correlação clínico-laboratorial.

Conclusão: A sífilis continua a ser um problema de saúde pública pelo que é necessário estabelecer programas de educação, rastreio e follow-up para reduzir a sua prevalência e tornar mais eficiente o rastreio dos parceiros.

Palavras-chave: Portugal; Sífilis/epidemiologia.

\section{ABSTRACT}

Introduction: Syphilis is a sexual and vertical transmitted disease. Its incidence is increasing in Europe, particularly, in Portugal. Material and Methods: A descriptive, retrospective study was performed based on positive treponemal tests from January to December 2013, at the Santa Maria Hospital, Lisbon. In-patients and out-patients evaluated in medical appointments and at the emergency department were included. We proceeded to epidemiological characterization, disease classification and definition of risk factors.

Results: We obtained a sample of 580 patients, of whom 51 with no clinical data and 45 with false positive serologies were excluded. There was a predominance of male patients (75\%) and a mean age of 47 years. Most (59\%) had syphilis successfully treated in the past and $3.7 \%$ were in follow-up. We recorded 13 primaries syphilis, 71 cases of secondary syphilis, 40 cases of early latent syphilis, 49 unknown duration syphilis and five cases of late latent syphilis. In the early syphilis group, $42 \%(n=124)$ were HIV-positive and, in $8 \%$ both diagnosis were done simultaneously.

Discussion: We emphasize the high prevalence of syphilis/HIV co-infection in patients with early syphilis, reinforcing the importance of promoting the use of preventive measures. We obtained $11 \%$ of patients with late clinical forms, which are notifiable since June 2014 , in Portugal. All serological tests for the diagnosis of syphilis have limitations which emphasizes the importance of clinical-laboratory correlation.

Conclusion: Syphilis remains an important public health problem. It is necessary to establish education programs, screening and follow-up strategies to reduce their prevalence and to perform more efficient screening of the partners.

Keywords: Portugal; Syphilis/epidemiology.

\section{INTRODUÇÃO}

A sífilis é uma doença infeciosa de transmissão sexual e vertical causada pela espiroqueta Treponema pallidum pallidum. As manifestações clínicas são polimorfas e incluem úlceras genitais, erupções cutâneas, linfadenopatias, atingimento cardiovascular e neurológico. A sua morbilidade é significativa, sobretudo na saúde materno-fetal. O diagnóstico é clínico e a confirmação é realizada por testes não treponémicos e treponémicos. O diagnóstico laboratorial da sífilis primária também pode ser obtido pela deteção de treponemas nos exsudados das lesões observadas em microscopia com fundo escuro ou por reação de polimerização em cadeia (PCR).

As estatísticas globais de sífilis revelam cerca de 10 milhões de novos casos cada ano. ${ }^{1} \mathrm{Na}$ Europa, a sua incidência está a aumentar, ${ }^{2}$ em particular, na comunidade MSM (men who have sex with men). Em Portugal, até 2013 apenas a sífilis precoce era de notificação obrigatória e, de acordo com os dados da Direção Geral de Saúde, entre 2011 e 2012 registou-se um aumento da sua incidência. ${ }^{3}$ A prevalência das outras formas clínicas é desconhecida

1. Serviço de Dermatologia. Hospital de Santa Maria. Centro Hospitalar Lisboa Norte. Lisboa. Portugal.

2. Serviço de Infeciologia. Hospital de Santa Maria. Centro Hospitalar Lisboa Norte. Lisboa. Portugal.

3. Serviço de Patologia Clínica. Hospital de Santa Maria. Centro Hospitalar Lisboa Norte. Lisboa. Portugal.

4. Unidade de Investigação em Dermatologia. Instituto de Medicina Molecular. Faculdade de Medicina. Universidade de Lisboa. Lisboa. Portugal.

5. Departamento de Microbiologia Médica. Instituto de Higiene e Medicina Tropical. Lisboa. Portugal.

$\triangle$ Autor correspondente: Leonor Lopes. leonorlopes@gmail.com

Recebido: 27 de janeiro de 2015 - Aceite: 03 de agosto de 2015 | Copyright @ Ordem dos Médicos 2016 
e a notificação não inclui outros dados importantes como a presença de coinfeções.

Os objetivos do estudo foram: identificar o número de casos de sífilis detetados num hospital público terciário na área de Lisboa, classificar as diferentes formas clínicas e analisar variáveis laboratoriais e demográficas associadas.

\section{MATERIAL E MÉTODOS}

Realizou-se um estudo retrospetivo e descritivo através da análise laboratorial dos testes treponémicos e não-treponémicos, realizados entre 1 de janeiro e 31 de dezembro de 2013, no Hospital de Santa Maria, em Lisboa. O rastreio, nesta unidade hospitalar, não é efetuado de forma universal mas apenas a pedido do médico assistente. No entanto, quando requisitado é seguido sempre o mesmo algoritmo diagnóstico. Procedeu-se à caraterização epidemiológica (sexo, idade e local de diagnóstico), classificação clínica e de fatores de risco associados [antecedentes de sífilis ou de outras infeções sexualmente transmissíveis (IST's)]. Os critérios de inclusão foram: idade igual ou superior a 16 anos e pelo menos um teste treponémico positivo [CLIA (chemiluminescence immunoassay) e/ou TPHA (treponema pallidum haemagglutination)], em utentes observados nas consultas de ambulatório, no hospital de dia, na urgência e no internamento. O estudo teve a aprovação da comissão de ética a 13 de novembro de 2013. Para a classificação das formas clínicas adotou-se os critérios dos Centers for Disease Control and Prevention (CDC), de acordo com a Tabela 1.

\section{RESULTADOS}

O número total de testes treponémicos realizados no Hospital de Santa Maria, no ano de 2013, foi 8 637. Destes, 580 doentes (amostra inicial) reuniam os critérios de inclusão. Foram excluídos da amostra 51 doentes por ausência de dados clínicos que permitissem classificar a forma clínica da doença e 45 por valores falsos positivos [CLIA po- sitivo; TPHA e fluorescent treponemal antibody absorption (FTA-abs) negativos)] (Fig. 1).

A amostra final foi constituída por 484 doentes, 363 (75\%) do sexo masculino e 121 (25\%) do feminino. Tinham idades compreendidas entre 16 e 86 anos e média etária de 46,6 \pm 14,7 anos.

Relativamente às formas clínicas, a maioria $(n=288)$ tinha serologias compatíveis com sífilis tratada no passado e 3,7\% encontrava-se em vigilância clínica, porque o diagnóstico foi efetuado no final de 2012 e tinham valores de VDRL $\geq 2$ dils. Foram identificados 124 sífilis precoce. $\mathrm{Na}$ Tabela 2 está ilustrada a classificação das diferentes formas clínicas de sífilis precoce.

Observou-se sífilis tardia em 54, sendo 49 latente de duração indeterminada e cinco latente tardia. Oito apresentavam sífilis secundária com envolvimento do sistema nervoso central (três casos de sífilis meningovascular, dois de sífilis ocular, dois de meningite e um de otite sifilítica). Não se registou sífilis terciária.

No grupo sífilis precoce, $42 \%(n=52)$ tinham antecedentes pessoais de infeção $\mathrm{VIH}$, já em seguimento em consultas externas e $33 \%(n=41)$ de sífilis. Identificaram-se dez ( $8 \%)$ novos casos de coinfeção sífilis/VIH. As restantes doenças de transmissão sexual identificadas foram: vírus do papiloma humano $(n=5)$, vírus da hepatite $C(n=3)$, herpes genital $(n=3)$, infeção a Chlamydia trachomatis $(n=3)$ e a Neisseria gonorrhoeae $(n=1)$. No grupo de doentes com secundarismo e neurosífilis recente, 27 doentes tinham antecedentes pessoais de $\mathrm{VIH}$ e, em oito foi realizado o diagnóstico inaugural desta infeção.

Os testes com resultados positivos foram requisitados no serviço de urgência em 73 casos, na consulta de ambulatório ou em hospital dia em 127 e no internamento em 284 $(0,7 \%$ do total de internamentos). Os doentes internados encontravam-se, essencialmente, nos Serviços de Medicina e de Infeciologia, e os do ambulatório eram provenientes do Hospital de Dia de Infeciologia e da Consulta Externa de

Tabela 1 - Classificação das formas clínicas de sífilis

\begin{tabular}{ll}
\hline Formas clínicas da doença & Definição \\
\hline Sífilis primária & $\begin{array}{l}\text { Presença de uma ou mais úlceras consistentes com o diagnóstico e um teste serológico reativo (VDRL } \\
\text { ou RPR, FTA-abs ou MHA-TP). }\end{array}$ \\
Sífilis secundária & $\begin{array}{l}\text { Lesões mucocutâneas difusas ou localizadas frequentemente acompanhadas de linfadenopatias } \\
\text { generalizadas. A úlcera primária pode ainda estar presente. Requer teste treponémico e não } \\
\text { treponémico reativos (VDRL ou RPR com título } \geq 4) .\end{array}$
\end{tabular}

Sífilis latente precoce

Quando a infeção ocorreu nos últimos 12 meses e está presente um ou mais dos seguintes critérios: Seroconversão documentada ou um teste não treponémico com um aumento do título $\geq 4$; história consistente com sífilis $1^{a}$ ou $2^{a}$ durante este período; história de exposição sexual a parceiro com sífilis precoce neste período; testes não treponémicos ou treponémicos positivos de um doente cuja exposição só ocorreu neste período.

Sífilis latente tardia

Quando a infeção ocorreu > 12 meses e não reúne critérios específicos para sífilis latente precoce. Necessita de um teste treponémico e um teste não-treponémico reactivos.

Sífilis latente de duração Não reúne critérios de sífilis latente precoce nem tardia, com teste treponémico e não treponémico indeterminada reativos.

MHA-TP: microhemagglutination assay for T. pallidum 


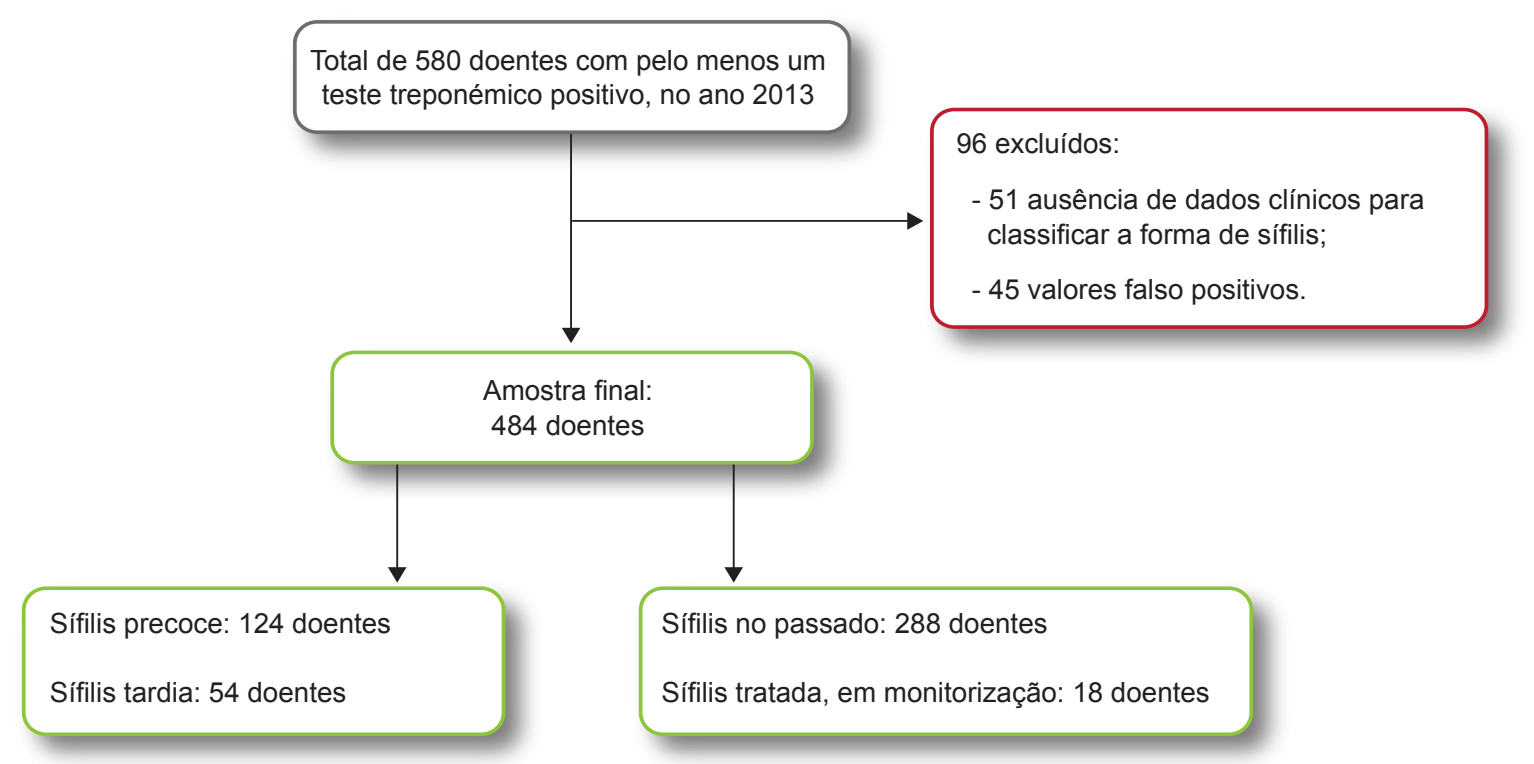

Figura 1 - Fluxograma do estudo

Dermatologia. No que se refere aos doentes com sífilis primária e secundária, o diagnóstico foi efetuado na urgência central em 61, na Consulta de Dermatologia e Infeciologia em dez cada, e os restantes (três casos) noutros serviços do hospital.

$\mathrm{Na}$ análise laboratorial verificou-se discordância entre valores de testes treponémicos (CLIA positivo e TPHA negativo) em sete doentes. Destes, cinco tinham antecedentes pessoais de sífilis e infeção VIH. No que se refere às formas clínicas, foram identificadas sífilis no passado $(n=4)$, sífilis latente precoce $(n=2)$ e sífilis latente de duração indeterminada $(n=1)$. Não se registaram casos de TPHA positivo e CLIA negativo (Tabela 3). O CLIA não foi requisitado em 90 doentes. Registou-se fenómeno prozona (obtenção de valor falso negativo, nos testes não-treponémicos, resultante de uma elevada concentração do título de anticorpos produzido pelo doente) em sete casos.

\section{DISCUSSÃO}

A sífilis, assim como outras doenças que causam ulceração genital, facilita a aquisição e transmissão do VIH, uma vez que altera a barreira cutâneo-mucosa e origina inflamação local. ${ }^{4,5}$ Além disso, a ativação da resposta imune em hospedeiros infetados contribui para o aumento da replicação viral. ${ }^{5,6} \mathrm{~A}$ coinfeção sífilis/VIH é bastante comum em MSM, atingindo prevalências que variam entre os $49 \%$ e os $75 \% .^{5}$

Em Portugal, Ferreira et $\mathrm{al}^{7}$ destacam a elevada percen-

Tabela 2 - Sífilis precoce: classificação das formas clínicas

\begin{tabular}{lc}
\hline Sífilis precoce $(\mathbf{n}=\mathbf{1 2 4})$ & $\mathbf{N}^{\circ}$ de doentes $(\%)$ \\
\hline Primária & $13(10,5)$ \\
Secundária & $71(57,3)$ \\
Latente precoce & $40(32,2)$ \\
\hline
\end{tabular}

tagem de doentes com VIH prévio que adquire sífilis recente $(70,4 \%)$ assim como outras IST's $(31,5 \%)$. Neste estudo, constatou-se tal facto em $50 \%$ dos portadores de sífilis precoce. Estas elevadas taxas são devido à persistência de comportamentos sexuais de risco e ao incumprimento de medidas preventivas na transmissão destas doenças. Desta forma, é aconselhado a realização do rastreio laboratorial em populações de risco (consumidores de estupefacientes, múltiplos parceiros sexuais, VIH e MSM), de forma anual ou sempre que o quadro clínico o justifique. Nestes doentes deve realizar-se também rastreio de outras IST's, nomeadamente, $\mathrm{VIH}$, hepatites $\mathrm{B}$ e $\mathrm{C}$ no sangue, pesquisa de Chlamydia trachomatis (por PCR) na urina e de Chlamydia trachomatis e Neisseria gonorrhoeae no exsudado uretral, se presente.

Nem todos os clínicos realizam de forma sistemática o rastreio de IST's, o que justifica o reduzido número de casos identificados nesta revisão. Para além da falta de uniformidade no rastreio, as principais limitações deste estudo foram a ausência de dados nos processos clínicos, que levou à exclusão de 51 doentes e a impossibilidade na obtenção de variáveis relevantes para programas de controlo de IST's como orientação sexual, etnia, número de parceiros e utilização consistente de medidas barreira durante a prática sexual.

Em 2009, houve uma alteração das normas europeias de rastreio da sífilis [guidelines da International Union against Sexually Transmitted Infections (IUSTI)].

Tabela 3 - Resultados dos testes treponémicos (CLIA e TPHA)

\begin{tabular}{cccc}
\hline & & \multicolumn{2}{c}{ TPHA } \\
& & Positivo & Negativo \\
\hline \multirow{2}{*}{ CLIA } & Positivo & 387 & 7 \\
& Negativo & 0 & Excluídos \\
\hline
\end{tabular}


Em populações de baixo risco, o rastreio deve iniciar-se por um teste treponémico (CLIA) ${ }^{8,9}$ Este é o método ideal para screening de populações assintomáticas com número elevado de doentes, uma vez que é rápido, automatizado, com elevada sensibilidade (95\% - 99\%) e especificidade (98\% - 99\%). ${ }^{9-11}$ Se for positivo, deve realizar-se outro teste treponémico (TPHA) para confirmação, e um não-treponémico (VDRL ou RPR), para monitorização (avaliação da eficácia terapêutica).

No estudo identificaram-se $8,9 \%$ de doentes com discordância entre testes treponémicos, dos quais sete foram incluídos e 45 foram excluídos por resultados falsos positivos. A exclusão deveu-se à inexistência de história clínica sugestiva de sífilis e, em populações de baixo risco, serem considerados falsos positivos se o CLIA for positivo e os restantes testes treponémicos e não-treponémicos negativos. ${ }^{9}$ No entanto, Ooi et al ${ }^{12}$ verificaram que, se tivessem sido realizadas histórias clínicas completas, 32\% tinham dados clínicos que aumentariam a suspeita desta patologia. ${ }^{12}$ Sete foram incluídos apesar da inconsistência dos testes treponémicos, dos quais dois com sífilis precoce e cinco com sífilis tratada no passado ou tardia. Estas divergências são mais frequentes nos portadores do VIH, o que está de acordo com os resultados apresentados.

Todos os testes serológicos para o diagnóstico de sífilis apresentam limitações. ${ }^{10}$ Nestas situações, a realização de história clínica pormenorizada, com ênfase nos dados epidemiológicos e a repetição adicional dos testes são úteis na interpretação dos resultados, o que enfatiza a importância da correlação clínico-laboratorial. ${ }^{10}$

Apesar da deteção de sífilis precoce ser a prioridade dos programas de rastreio, no Reino Unido a maioria dos doentes recém-diagnosticados tem infeção tardia. ${ }^{11}$ Neste estudo, registaram-se $11 \%$ de casos. Por isso, salienta-se a importância do rastreio sistemático a todos os doentes in- ternados de forma a identificar esta forma clínica da doença. Desde 16 de junho de 2014, de acordo com o despacho $n .^{\circ} 609-A / 2014$, a sífilis tardia passou também a ser de notificação obrigatória em Portugal, o que futuramente irá revelar a real dimensão deste problema.

\section{CONCLUSÃO}

A falta de recursos na promoção da educação sexual, o baixo nível de consciencialização em saúde, assim como a presença de outras IST's aumentam o risco de aquisição e transmissão de sífilis. No século XXI, esta doença continua a ser um problema de saúde pública, pelo que é necessário estabelecer programas de educação, rastreio e follow-up para reduzir a sua prevalência e tornar mais eficiente o rastreio dos parceiros.

\section{PROTECÇÃO DE PESSOAS E ANIMAIS}

Os autores declaram que os procedimentos seguidos estavam de acordo com os regulamentos estabelecidos pelos responsáveis da Comissão de Investigação Clínica e Ética e de acordo com a Declaração de Helsínquia da Associação Médica Mundial.

\section{CONFIDENCIALIDADE DOS DADOS}

Os autores declaram ter seguido os protocolos do seu centro de trabalho acerca da publicação dos dados de doentes.

\section{CONFLITOS DE INTERESSE}

Os autores declaram não ter conflitos de interesse relativamente ao presente artigo.

\section{FONTES DE FINANCIAMENTO}

Não existiram fontes externas de financiamento para a realização deste artigo.

\section{REFERÊNCIAS}

1. Tong ML, Lin LR, Liu GL, Zhang HL, Zeng YL, Zheng WH, et al. Factors associated with serological cure and the serofast state of HIV-negative patients with primary, secondary, latent, and tertiary syphilis. PLoS One. 2013;8:e70102.

2. ECDC (2013) - Surveillance Report: Annual epidemiological report Reporting on 2011 surveillance data and 2012 epidemic intelligence data. [consultado 2015 jan 12]. Disponível em: http://www.ecdc.europa. eu/en/publications/Publications/Annual-Epidemiological-Report-2013. pdf.

3. Direção-Geral da Saúde. Doenças de declaração obrigatória 20092012 - Volume I. [consultado 2015 jan 12]. Disponível em: http:// www.google.pt/url?sa $=t \& r c t=j \& q=\& e s r c=s \&$ source $=$ web $\& c d=2 \&$ ved=0CCMQFjAB\&url=http $\% 3 A \% 2 F \% 2 F w w w . d g s . p t \% 2 F p o r t a l-$ da-estatistica-da-saude $\% 2 F$ publicacoes-estatisticas-146417-pdf. aspx\%3Fv\%3D11736b14-73e6-4b34-a8e8-d22502108547\&ei=h M7HVJ2qMpaZsQT2ooCoCQ\&usg=AFQjCNErfKOuiE2ON9cHIJcUr8BolBgZw\&bvm=bv.84349003,d.cWc.

4. Council of State and Territorial Epidemiologists. Recent changes to gonorrhea and syphilis case definitions: program impact. [consultado 2015 jan 12]. Disponível em: http://www.cdc.gov/std/stats/casedefprogramimpact-2014.pdf.

5. Repiso B, Frieyro M, Rivas-Ruiz F, De Troya M. Condom use and number of sexual partners among male syphilis patients who report

having sex with men. Actas Dermosifiliogr. 2010;101:847-52.

6. Zetola NM, Engelman J, Jensen TP, Klausner JD. Syphilis in the United States: an update for clinicians with an emphasis on HIV coinfection. Mayo Clin Proc. 2007;82:1091-102.

7. Ferreira O, Lisboa C, Ramos FM, Azevedo F. Sífilis numa consulta de infecções sexualmente transmissíveis - análise de 880 doentes. Rev Soc Port Dermatol Venereol. 2012;70:99-104

8. Janier M, Hegyi V, Dupin N, Unemo M, Tiplica GS, Potocnik M, et al. European Guideline on the management of syphilis. Eur Acad Dermatol Venereol. 2015;29:1248.

9. Young H, Moyes A, Seagar L, McMillan A. Novel recombinant-antigen enzyme immunoassay for serological diagnosis of syphilis. J Clin Microbiol. 1998;36:913-7.

10. Park IU, Chow JM, Bolan G, Stanley M, Shieh J, Schapiro JM. Screening for syphilis with the treponemal immunoassay: analysis of discordant serology results and implications for clinical management. Infect J Dis. 2011;204:1297-304.

11. Young H. Guidelines for serological testing for syphilis. Sex Transm Infect. 2000;76:403-5.

12. Ooi C, Robertson $P$, Donovan B. Investigation of isolated positive syphilis enzyme immunoassay (ICE Murex) results. Int J STD AIDS 2002;13:761-4 